\title{
Coherent Control of Reactive Scattering
}

\author{
Alexander Abrashkevich, Moshe Shapiro, * and Paul Brumer \\ Chemical Physics Theory Group, University of Toronto, and Photonics Research Ontario, Toronto, Canada M5S 3 H6
}

(Received 11 December 1997)

\begin{abstract}
Coherent control of bimolecular reactions is demonstrated for 3D atom-diatom reactive scattering. In particular, a superposition of initial degenerate $(v j k)$ diatomic states is used to control reactive integral and differential cross sections in $\mathrm{D}+\mathrm{H}_{2}(v j k) \rightarrow \mathrm{H}+\mathrm{HD}$, where $v$ and $j$ are the vibrational and rotational quantum numbers of a diatom and $k$ is the projection quantum number of the diatom angular momentum onto the initial relative translational velocity vector. Control over the ratio of reactive to nonreactive scattering is extensive. [S0031-9007(98)07470-5]
\end{abstract}

PACS numbers: 82.40.Dm, 34.50.Lf, 34.50.Rk

Controlling the dynamics of atoms and molecules has been a long-standing goal in physics. A recent approach, called coherent control, offers a systematic route to reaching this goal. Specifically, in coherent control one uses the quantum properties of light and matter to introduce quantum interference terms into the system dynamics; these terms, and hence the dynamical outcome, can be altered by manipulating laboratory parameters. This approach has proven successful, both theoretically and experimentally, in controlling the outcome of unimolecular processes such as photodissociation (see, e.g., [1-4]). By contrast, control over collisional processes has presented a much greater challenge [5].

In this paper we show that extensive control over quantum reactive scattering can indeed be attained by scattering from an initially prepared superposition of degenerate diatomic states, demonstrating the essence of coherent control in a fundamental collisional process. This constitutes a major extension of the range of possible applications of coherent control. In particular, we show, using the results of three-dimensional calculations for $\mathrm{D}+\mathrm{H}_{2} \rightarrow \mathrm{H}+\mathrm{HD}$ on a realistic potential surface, that reactive vs nonreactive cross sections (both differential and integral) can be extensively controlled by varying phases and amplitudes in the initially prepared superposition state. Furthermore, the proposed approach is easily extended to any nonrelativistic scattering problem (e.g., atom-atom scattering, low energy nuclear scattering, etc.).

Consider the reaction $A+B C \rightarrow B+A C$ or $C+A B$, where we label the three possible arrangement channels by the symbol $\alpha=(A+B C), b(B+A C)$, or $c(C+$ $A B)$. We consider the simplest method of introducing interference effects into dynamics by examining scattering from an asymptotic state $|\mathbf{n}, \alpha\rangle$ which is an energetically degenerate superposition of scattering states composed of two degenerate states of the diatom. The extension to include more states is straightforward. Consider then

$$
|\mathbf{n}, \alpha\rangle=\sum_{i=1,2} a_{i}\left|\alpha v j k_{i}\right\rangle\left|E_{\alpha}^{\mathrm{kin}}(i)\right\rangle\left|E_{\mathrm{cm}}(i)\right\rangle,
$$

where $\mathbf{n}$ encapsulates all the state labels other than $\alpha$, $\left|E_{\alpha}^{\mathrm{kin}}(i)\right\rangle$ are plane waves describing the free motion of the atom relative to the diatom for the $\alpha$ arrangement and $\left|E_{\mathrm{cm}}(i)\right\rangle$ describes the motion of the atom-diatom center of mass. The superposition state $|\mathbf{n}, \alpha\rangle$, assumed normalized, is therefore composed of two degenerate eigenstates $\left|\alpha v j k_{i}\right\rangle\left|E_{\alpha}^{\mathrm{kin}}(i)\right\rangle$ of the asymptotic $\alpha$-channel Hamiltonian, where the quantum numbers $v, j$, and $k_{i}$ denote the vibrational, rotational, and angular momentum projection quantum numbers of the diatomic. The latter, $k_{i}$, is taken as the helicity, i.e., the angular momentum projection along the relative initial translational velocity vector, and the diatom states $\left|\alpha v j k_{i}\right\rangle$ are of energy $\epsilon_{\alpha v j}$.

Traditional time independent scattering theory deals with asymptotic states where one of the $a_{i}=0$, so that scattering correlates with one asymptotic state. The equations below are a direct, but significant, extension of these traditional results. In particular, the differential cross section for forming the $\alpha^{\prime}$ arrangement at scattering angle $\theta$, having started from the $|\mathbf{n}, \alpha\rangle$ superposition state [Eq. (1)], is given by

$$
\sigma^{R}(\theta)=\sum_{v^{\prime}, j^{\prime}, k^{\prime}}\left|\sum_{m=1,2}\left\langle E_{\mathrm{cm}}(m)\left|\sum_{i=1,2} a_{i} f_{\alpha^{\prime} v^{\prime} j^{\prime} k^{\prime} \leftarrow \alpha v j k_{i}}(\pi-\theta)\right| E_{\mathrm{cm}}(i)\right\rangle\right|^{2},
$$

where the superscript $R$ denotes reactive scattering into a specific final arrangement channel $\alpha^{\prime} \neq \alpha$ and where the scattering amplitude is

$$
\begin{aligned}
f_{\alpha^{\prime} v^{\prime} j^{\prime} k^{\prime} \leftarrow \alpha v j k_{i}}(\theta)= & \left(2 i \mathrm{k}_{\alpha v j}\right)^{-1} \sum_{J}(2 J+1) d_{k^{\prime} k_{i}}^{J}(\theta) \\
& \times\left[S_{\alpha^{\prime} v^{\prime} j^{\prime} k^{\prime}, \alpha v j k_{i}}^{J}-\delta_{\alpha^{\prime} \alpha} \delta_{v^{\prime} v} \delta_{j^{\prime} j} \delta_{k^{\prime} k_{i}}\right] .
\end{aligned}
$$

Here $S_{\alpha^{\prime} v^{\prime} j^{\prime} k^{\prime}, \alpha v j k_{i}}^{J}$ are the elements of scattering $S$ matrix in the helicity representation, $J$ is the total angular momentum, $k^{\prime}$ is the helicity of the product diatom (i.e., the projection of the diatom angular momentum onto the final relative translational velocity vector), $d_{k^{\prime} k_{i}}^{J}(\theta)$ are the reduced rotation matrices [6], and $\mathrm{k}_{\alpha v j}=\sqrt{2 \mu_{\alpha}\left(E-\epsilon_{\alpha v j}\right)} / \hbar$, with $\mu_{\alpha}$ being the 
atom-diatom reduced mass in the $\alpha$ channel. Expanding the square in Eq. (2) gives the reactive differential scattering cross section as

$\sigma^{R}(\theta)=\left|a_{1}\right|^{2} \sigma_{11}^{R}(\theta)+\left|a_{2}\right|^{2} \sigma_{22}^{R}(\theta)+2 \operatorname{Re}\left\{a_{1}^{*} a_{2} \sigma_{12}^{R}(\theta)\right\}$,

where

$$
\sigma_{i i}^{R}(\theta)=\sum_{v^{\prime}, j^{\prime}, k^{\prime}}\left|f_{\alpha^{\prime} v^{\prime} j^{\prime} k^{\prime} \leftarrow \alpha v j k_{i}}(\pi-\theta)\right|^{2}, \quad i=1,2,
$$

$$
\begin{aligned}
\sigma_{12}^{R}(\theta)= & \sum_{v^{\prime}, j^{\prime}, k^{\prime}}\left\langle E_{\mathrm{cm}}(1) \mid E_{\mathrm{cm}}(2)\right\rangle_{V} f_{\alpha^{\prime} v^{\prime} j^{\prime} k^{\prime} \leftarrow \alpha v j k_{1}}(\pi-\theta) \\
& \times f_{\alpha^{\prime} v^{\prime} j^{\prime} k^{\prime} \leftarrow \alpha v j k_{2}}^{*}(\pi-\theta) .
\end{aligned}
$$

In the cases considered here, i.e., a superposition of degenerate diatomic states, the overlap $\left\langle E_{\mathrm{cm}}(1) \mid E_{\mathrm{cm}}(2)\right\rangle_{V}$ of the center of mass wave functions over the scattering volume $V$, which appears in Eq. (6), is unity.

Integration of Eq. (4) over angle $\theta$ gives the integral reactive cross section $\sigma^{R}$. This can also be written as three terms, as in Eq. (4), but with $\sigma_{i j}^{R}(\theta)$ replaced by $\sigma_{i j}^{R}$ where

$$
\begin{gathered}
\sigma_{i i}^{R}=\frac{\pi}{\mathrm{k}_{\alpha v j}^{2}} \sum_{v^{\prime}, j^{\prime}, k^{\prime}} \sum_{J}(2 J+1)\left|S_{\alpha^{\prime} v^{\prime} j^{\prime} k^{\prime}, \alpha v j k_{i}}^{J}\right|^{2}, \quad i=1,2, \\
\sigma_{12}^{R}=\frac{\pi}{2 \mathrm{k}_{\alpha v j}^{2}}\left\langle E_{\mathrm{cm}}(1) \mid E_{\mathrm{cm}}(2)\right\rangle_{V} \sum_{v^{\prime}, j^{\prime}, k^{\prime} J, J^{\prime}} \sum_{0}(2 J+1)\left(2 J^{\prime}+1\right) S_{\alpha^{\prime} v^{\prime} j^{\prime} k^{\prime}, \alpha v j k_{1}}^{J}\left[S_{\alpha^{\prime} v^{\prime} j^{\prime} k^{\prime}, \alpha v j k_{2}}^{J^{\prime}}\right]^{*} \\
\times \int_{0}^{\pi} d \theta \sin \theta d_{k^{\prime} k_{1}}^{J}(\pi-\theta) d_{k^{\prime} k_{2}}^{J^{\prime}}(\pi-\theta), \quad \alpha \neq \alpha^{\prime} .
\end{gathered}
$$

Note that $\sigma_{i i}^{R}(\theta)$ and $\sigma_{i i}^{R}$ in Eqs. (5) and (7) are the differential and integral cross sections that appear in standard scattering theory, while $\sigma_{12}^{R}(\theta)$ and $\sigma_{12}^{R}$ in Eqs. (6) and (8) are new types of interference terms which allow for control through the $a_{i}$ over the atom-diatom collision process. Note further that significant control requires substantial $\sigma_{12}^{R}$ and, by the Schwartz inequality $\left[\left|\sigma_{12}^{R}\right| \leq \sqrt{\sigma_{11}^{R} \sigma_{22}^{R}}\right]$, large $\sigma_{11}^{R}$ and $\sigma_{22}^{R}$. Significantly then, extensive control is not limited to regions near the reactive threshold [7]. Further, although Eqs. (4), (7), and (8) indicate that the differential and total reactive cross sections are controllable, removing the sums over $v^{\prime}, j^{\prime}$, and $k^{\prime}$ shows that detailed cross sections to product states $\left|\alpha^{\prime}, v^{\prime}, j^{\prime}, k^{\prime}\right\rangle$ can also be controlled.

Thus, by varying the coefficients $a_{i}$ in Eq. (1) through an initial preparation step, we can directly alter the interference term $\sigma_{12}^{R}$ and hence control the scattering cross sections. Such a preparation might be carried out, for example, by a suitably devised molecular beam experiment where the diatomic is excited, via elliptically polarized light to a collection of well defined $m_{j}$ states. Alternatively, the prereactive step may consist of coherently controlling the photodissociation of a polyatomic molecule [1] to produce the diatom in a controlled superposition of $k$ states relative to an incoming scattering partner. For example, in the $\mathrm{D}+\mathrm{H}_{2}$ case considered below we can subject $\mathrm{H}_{2} \mathrm{~S}$ to a coherently controlled preparatory step producing $\mathrm{H}_{2}$. Aiming the $\mathrm{D}$ atom exactly antiparallel to the direction of motion of the $\mathrm{H}_{2}$ will then produce the desired scattering of a $k$ superposition.

To examine the extent of control it is useful to rewrite the total reactive cross section in the form

$$
\begin{aligned}
\sigma^{R}= & {\left[\sigma_{11}^{R}+x^{2} \sigma_{22}^{R}+2 x\left|\sigma_{12}^{R}\right|\right.} \\
& \left.\times \cos \left(\delta_{12}^{R}+\phi_{12}\right)\right] /\left(1+x^{2}\right),
\end{aligned}
$$

where $\quad x=\left|a_{2} / a_{1}\right|, \quad \phi_{12}=\arg \left(a_{2} / a_{1}\right), \quad$ and $\quad \delta_{12}^{R}=$ $\arg \left(\sigma_{12}^{R}\right)$. Of greatest interest is control over the reactive to nonreactive cross section branching ratio which is given by

$$
\frac{\sigma^{R}}{\sigma^{\mathrm{NR}}}=\frac{\sigma_{11}^{R}+x^{2} \sigma_{22}^{R}+2 x\left|\sigma_{12}^{R}\right| \cos \left(\delta_{12}^{R}+\phi_{12}\right)}{\sigma_{11}^{\mathrm{NR}}+x^{2} \sigma_{22}^{\mathrm{NR}}+2 x\left|\sigma_{12}^{\mathrm{NR}}\right| \cos \left(\delta_{12}^{\mathrm{NR}}+\phi_{12}\right)},
$$

where the nonreactive terms are denoted NR and have definitions analogous to their reactive counterparts (i.e., $\alpha^{\prime}$ is replaced by $\alpha$ in the defining equations for the cross sections). A formula similar to Eq. (10) holds for the ratio of reactive to nonreactive differential cross sections with total cross section terms replaced by differential cross section terms; that is, with $\sigma_{m n}^{R}$ replaced by $\sigma_{m n}^{R}(\theta)$, etc.

In this Letter we apply this approach to $\mathrm{D}+\mathrm{H}_{2} \rightarrow$ $\mathrm{H}+\mathrm{HD}$ at $E=1.25 \mathrm{eV}$. In particular, we expose the dependence of the cross sections on $a_{i}$. Scattering calculations were done with the log-derivative version of the Kohn variational principle [8] using a basis set contraction approach [9], and cross sections were obtained via the symmetrization procedure described in Ref. [10]. Calculations were carried out using the accurate LSTH (Liu-Siegbahn-Truhlar-Horowitz) [11] potential energy surface for total angular momentum from $J=0$ to 31 with $j_{\max }=14$, ensuring fully converged cross sections for the chosen energy. It is worth noting that these calculations are CPU intensive, requiring in excess of 35 hours of CRAY T-90 time. Calculation of scattering amplitudes for all possible transitions took about 90 hours of the SGI PCA CPU time.

Control results for a specific initial vibrotational state $(v=0, j=2)$ of diatom $\mathrm{H}_{2}$ at scattering energy $E=$ $1.25 \mathrm{eV}$ are discussed below. (Qualitatively similar results were obtained for other initial states and for D + $\mathrm{H}_{2} \rightarrow \mathrm{H}+\mathrm{HD}$ and $\mathrm{H}+\mathrm{D}_{2} \rightarrow \mathrm{D}+\mathrm{HD}$ [12].) This energy, rather far from the reaction threshold, allows us 
to demonstrate substantial control in the presence of a significant natural reactive cross section.

Consider first the range of coherent control over the differential cross sections. Initial numerical tests showed

(a)

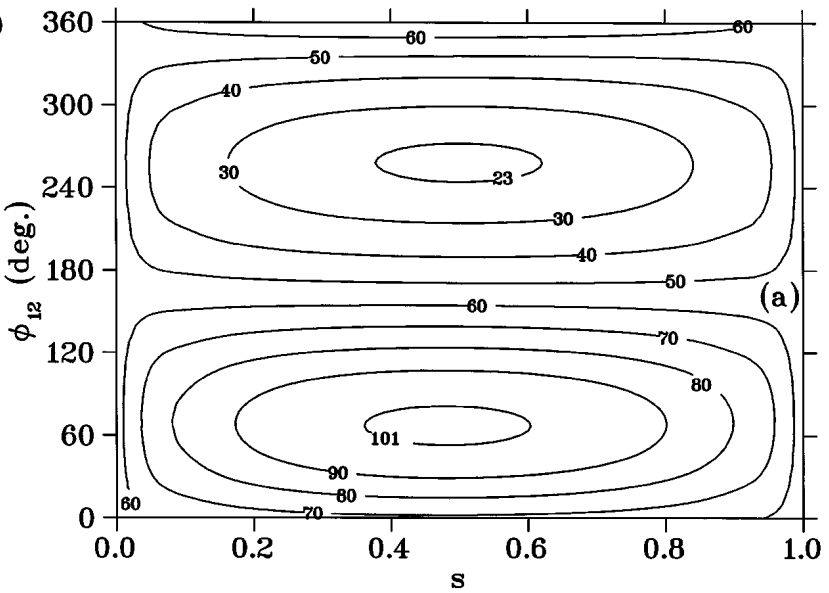

(b)

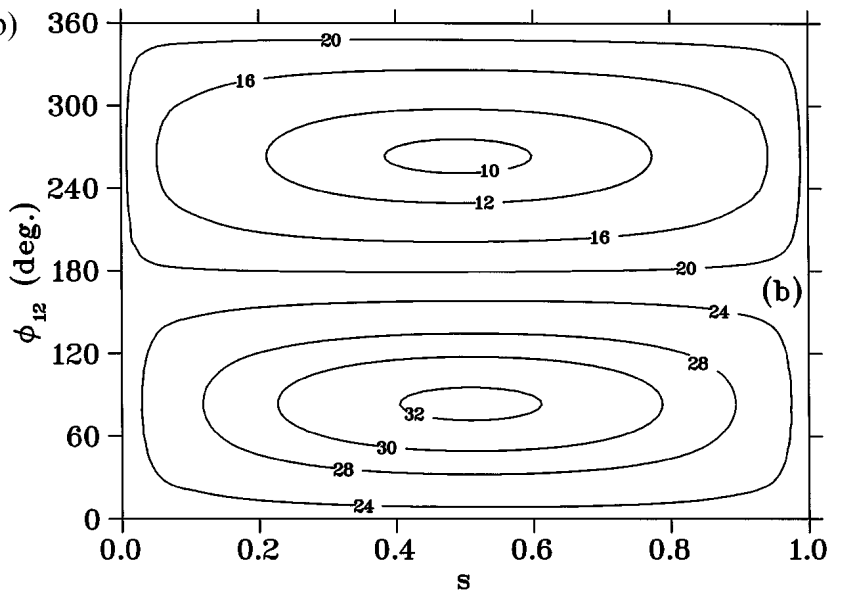

(c)

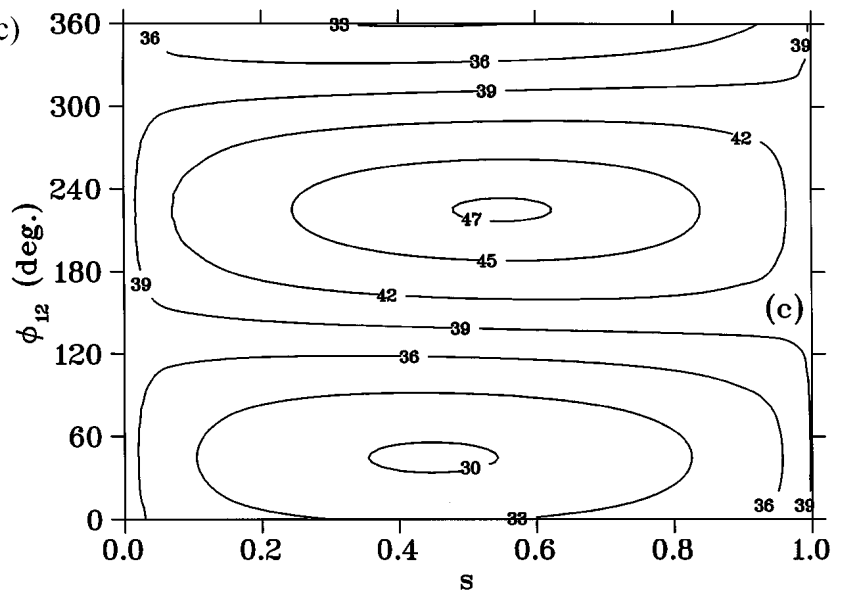

FIG. 1. Contour plot of the differential cross section as a function of $\phi_{12}$ and $s$, for a transition from an initial superposition state with $v=0, j=2, k_{1}=1, k_{2}=0$ at scattering angle $\theta=60^{\circ}$ and total energy $E=1.25 \mathrm{eV}$ : (a) Reactive to nonreactive ratio $\sigma^{R}(\theta) / \sigma^{\mathrm{NR}}(\theta)$, (b) reactive $\sigma^{R}(\theta)$, and (c) nonreactive $\sigma^{\mathrm{NR}}(\theta)$. Contour values in (a), (b), and (c) report values of $\sigma \times 10^{2}$. that the best control over the $\sigma^{R}(\theta) / \sigma^{\mathrm{NR}}(\theta)$ differential cross section ratio for transitions from initial states with $v=0, j=2$, and $k_{1}, k_{2}$ in the range $-2,-1, \ldots, 2$ occurred at $\theta \sim 60^{\circ}$. Figure 1a shows results for this ratio and scattering angle as a function of relative phase $\phi_{12}$ and the amplitude parameter $s=x^{2} /\left(1+x^{2}\right)$, for $k_{1}=1, k_{2}=0$. Varying $s$ from zero to one corresponds to changing the initial superposition from scattering out of state one ( $s=0$, corresponding to $\left.a_{1}=1, a_{2}=0\right)$ to scattering out of the second state $(s=1$ corresponding to $\left.a_{1}=0, a_{2}=1\right)$. The results clearly show substantial control over $\sigma^{R}(\theta) / \sigma^{\mathrm{NR}}(\theta)$. That is, varying $s$ and $\phi_{12}$ allows a change in the ratio from 0.23 to 1.01 , compared with the uncontrolled ratio of $\sim 0.55$. Similarly, for example, the $\sigma^{R}(\theta) / \sigma^{\mathrm{NR}}(\theta)$ ratio can be increased by a factor of 4.4 just by changing the phase angle $\phi_{12}$ from $260^{\circ}$ to $67^{\circ}$ at $s=0.49$.

Figures $1 \mathrm{~b}$ and $1 \mathrm{c}$ show the $\sigma^{R}$ and $\sigma^{\mathrm{NR}}$ corresponding to Fig. 1a. Control over both $\sigma^{R}\left(\theta=60^{\circ}\right)$ and $\sigma^{\mathrm{NR}}(\theta=$ $\left.60^{\circ}\right)$ is clearly seen, with $\sigma^{R}$ ranging from $0.10 a_{0}^{2} \mathrm{sr}^{-1}$ to $0.32 a_{0}^{2} \mathrm{sr}^{-1}$ as $s$ and $\phi_{12}$ are varied. This compares, for example, to the uncontrolled values of $\sigma_{11}^{R}(\theta)=0.21 \mathrm{sr}^{-1}$ and $\sigma_{22}^{R}(\theta)=0.22 a_{0}^{2} \mathrm{sr}^{-1}$ at $s=0$ and $s=1$. Similar control can be seen in $\sigma^{\mathrm{NR}}$. Indeed, both the constructive enhancement of $\sigma^{R}$ and the destructive depletion of $\sigma^{\mathrm{NR}}$ are seen to be responsible for the controlled $\sigma^{R} / \sigma^{\mathrm{NR}}$ maximum.

Also of interest is the $\theta$ dependence of $\sigma^{R}(\theta) / \sigma^{\mathrm{NR}}(\theta)$ as a function of $s$ and $\phi_{12}$. Figure 2 shows the $\theta$ dependence of this ratio, for scattering from a superposition state composed of $v=0, j=2, k_{1}=2, k_{2}=0$ at $\phi_{12}=157^{\circ}$ and at four different values of $s$. [Note that these results also show, for limits $s=0$ and $s=1$, the dependence of the ratio on the initial $k$ state, the first such data of its kind. That is, $s=0$ corresponds to scattering out of the initial state $(v=0, j=2, k=2)$, and $s=1$ corresponds to scattering from $(v=0, j=2$,

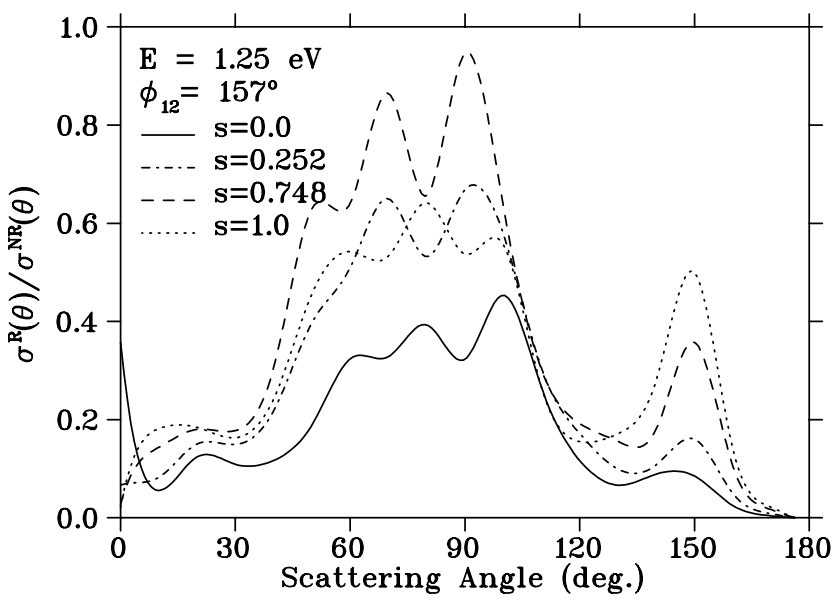

FIG. 2. Dependence of the $\sigma^{R}(\theta) / \sigma^{\mathrm{NR}}(\theta)$ ratio on the scattering angle $\theta$ at $\phi_{12}^{\max }=157^{\circ}$ and at four values of $s: s=0$, $s=1, s=0.252$, and $s=0.748$ for the case of $v=0$, $j=2, k_{1}=2, k_{2}=0$. 


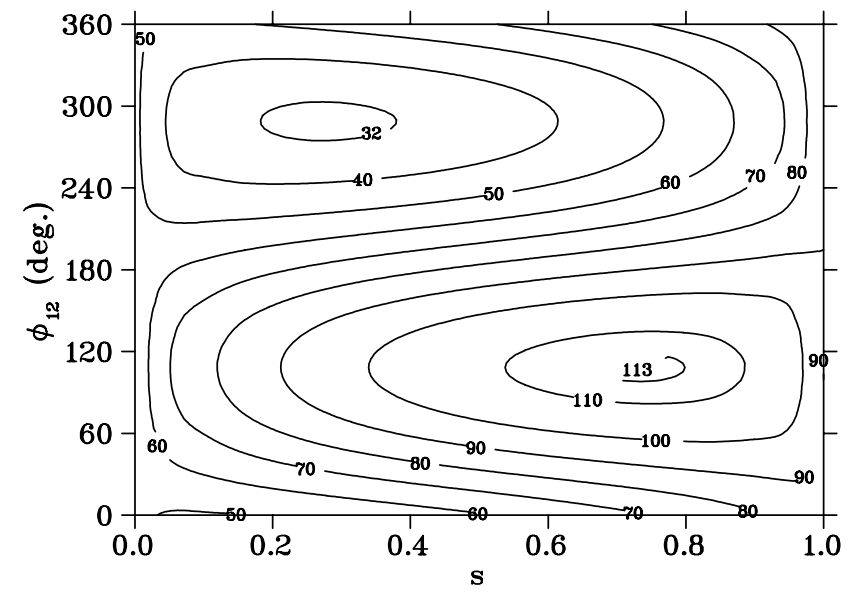

FIG. 3. Contour plot of the $\sigma^{R} / \sigma^{\mathrm{NR}}\left(\times 10^{3}\right)$ integral cross section ratio as a function of $\phi_{12}$ and $s$; for the case of $v=0, j=2, k_{1}=2, k_{2}=1$.

$k=0)$.] For this transition the controlled ratio of differential cross sections is seen to be considerably different from the uncontrolled ratio. For example, the controlled $\sigma^{R}(\theta) / \sigma^{\mathrm{NR}}(\theta)$ for $s=0.748$ is about twice as large as the uncontrolled ratios at $\theta=91^{\circ}$. Analysis of Fig. 2 shows that maxima and minima of the controlled $\sigma^{R} / \sigma^{\mathrm{NR}}$ ratio in the region between $50^{\circ}$ and $120^{\circ}$ are achieved at the corresponding minima and maxima of uncontrolled ratios. That is, quantum interference leads to constructive enhancement of the $\sigma^{R} / \sigma^{\mathrm{NR}}$ ratio at minima of uncontrolled ratios and to destructive depletion of controlled ratios at their maxima. Exactly the opposite behavior is observed in the outer $\theta$ regions. Hence coherent control changes both the magnitude and the structure of the differential cross section. Note also that the maxima of the controlled differential cross sections far exceed those for $s=0$ and $s=1$, confirming that the changes are due to quantum interference effects, as opposed to an interpolation between the $s=0$ and $s=1$ curves.

Of great interest as well is the extent of control over the integral cross sections. Figure 3 shows a contour plot of the ratio of integral cross sections $\sigma^{R} / \sigma^{\mathrm{NR}}$ for scattering from $v=0, j=2, k_{1}=2, k_{2}=1$ as a function of the control parameters $\phi_{12}$ and $s$. The ratio is seen to vary from 0.032 to 0.113 , showing maxima and minima that are well outside the range of the results for scattering from a single $k_{i}$ state. Greater control is anticipated at higher energies where the uncontrolled $\sigma^{R}$ and $\sigma^{\mathrm{NR}}$ become comparable.

In this Letter we have demonstrated that one may obtain considerable control over differential and integral cross sections branching ratios for realistic atom-diatom reactive scattering by preparing, and varying the characteristics of, a superposition of degenerate scattering states. Further, although not explored here, we expect similar control over detailed cross sections to specific product states.

Since the results of molecular beam experiments are well represented by time independent scattering theory and since our computations are state of the art, we anticipate that observed results will be in accord with our computations. Further, additional computations suggest that the control is not very sensitive to small changes in total energy so that one would anticipate little effect due to velocity distributions, etc. However, control is always sensitive to dephasing effects, e.g., external collisions, a feature to be examined in future papers.

This study opens a vast new area of application for coherent control. Work currently underway will extend studies to atom-heteronuclear diatom scattering to results at higher collision energies [12] and will incorporate specific scenarios to prepare the initial superposition state.

We thank Dr. B. Ramachandran for providing us with the REACT scattering code and for useful comments on its use. This work was supported in part by the U.S. Office of Naval Research, Photonics Research Ontario and by a grant of HPC time on the CRAY C-90 and the SGI Origin 2000 from the DoD ASC MSRC Center, and the CRAY T-90 and the SGI PCA from the DoD NAVOCEANO MSRC Center.

*Permanent address: Department of Chemical Physics, The Weizmann Institute of Science, 76100, Rehovot, Israel.

[1] M. Shapiro and P. Brumer, Trans. Faraday Soc. 93, 1263 (1997); P. Brumer and M. Shapiro, Annu. Rev. Phys. Chem. 43, 257 (1992); A. Shnitman, I. Sofer, I. Golub, A. Yogev, M. Shapiro, Z. Chen, and P. Brumer, Phys. Rev. Lett. 76, 2886 (1996).

[2] B. Kohler, J.L. Krause, F. Raski, K. R. Wilson, V. V. Yakovler, R. M. Whitnell, and Y. Yan, Acc. Chem. Res. 28, 133 (1995); W.S. Warren, H. Rabitz, and M. Dahleh, Science 259, 1581 (1993); S. A. Rice, ibid. 258, 412 (1992).

[3] B. Sheeny, B. Walker, and L. F. DiMauro, Phys. Rev. Lett. 74, 4799 (1995).

[4] E. Dupont, P. B. Corkum, H.C. Liu, M. Buchanan, and Z. R. Wasilewski, Phys. Rev. Lett. 74, 3596 (1995).

[5] M. Shapiro and P. Brumer, Phys. Rev. Lett. 77, 2574 (1996); D. Holmes, M. Shapiro, and P. Brumer, J. Chem. Phys. 105, 9162 (1996).

[6] W.H. Miller, J. Chem. Phys. 50, 407 (1969).

[7] J. L. Krause, M. Shapiro, and P. Brumer, J. Chem. Phys. 92, 1126 (1990).

[8] D. E. Manolopoulos and R.E. Wyatt, Chem. Phys. Lett. 152, 23 (1988).

[9] D.E. Manolopoulos, M. D'Mello, and R.E. Wyatt, J. Chem. Phys. 93, 403 (1990).

[10] J.Z.H. Zhang and W.H. Miller, J. Chem. Phys. 91, 1528 (1989).

[11] P. Siegbahn and B. Liu, J. Chem. Phys. 68, 2457 (1978); D. G. Truhlar and C. J. Horowitz, ibid. 68, 2466 (1978); 71, 1514 (1979).

[12] A. Abrashkevich, M. Shapiro, and P. Brumer (to be published). 